\title{
Pseudo-Differential Operators on a Half-Line
}

\section{H. O. CORDES}

\author{
Communicated by Hans LEWY
}

In [4], jointly with E. Herman, the author was discussing an algebra $\mathfrak{P}$ of singular integral operators on a half-line $0<x<\infty$ which allowed definition of a symbol different from the conventional one.

The symbol space topologically was a circle, with a continuum of points lying over the two boundary points 0 and $\infty$ of $(0, \infty)$ each.

As usual, an operator in $\mathfrak{B}$ is Fredholm if and only if its symbol does not vanish; an index-formula was derived.

In the present paper we first study an algebra $\mathfrak{A}$ of singular integral operators over the Hilbert space $\mathfrak{S}=\mathfrak{L}^{2}(0, \infty)$ which is generated from 3 of its commutative subalgebras:

a) The Wiener-Hopf-convolutions $H_{\varphi}^{*}$ with even $\mathcal{L}^{1}$-kernel defined as in (1.17).

b) An algebra $\mathfrak{M}$ of convolutions of $(0, \infty)$, considered as a topological group under multiplication, called Mellin-algebra, because its operators are diagonalized by the Mellin-transform.

c) The algebra $B$ of multiplications by a continuous function over $[0, \infty]$.

$\mathfrak{A}$ contains the algebra $\mathfrak{P}$ as a $C^{*}$-sub-algebra.

Again we achieve precise control over the Fredholm properties of operators in $\mathfrak{A}$ by defining a symbol whose non-vanishing is a necessary and sufficient condition for the operator to be Fredholm, and by deriving an index formula.

This new larger algebra $\mathfrak{A}$ will serve two purposes: a) It now can be used to define a class of pseudo-differential operators over $(0, \infty)$, which then especially will contain differential operators defined with boundary conditions; b) One of the sub-algebras of $\mathfrak{A}$ (called $\mathfrak{F}$ ) will play a key part in a paper to appear [3], where we attempt the same investigation of pseudo-differential operators involving boundary conditions for a half-space.

1. The Wiener-Hopf convolutions. Conventionally for $\varphi(s), \psi(s) \varepsilon \mathcal{L}^{1}(\mathbf{R})$ the convolution product is defined by

$$
\varphi * \psi=(2 \pi)^{-1 / 2} \int_{-\infty}^{+\infty} \varphi(x-y) \psi(y) d y .
$$


Then if $\hat{\varphi}=F \varphi$, defined by

$$
\hat{\varphi}(\xi)=(2 \pi)^{-1 / 2} \int_{-\infty}^{+\infty} \varphi(x) e^{-i x \xi} d x
$$

denotes the Fourier transform of $\varphi$ (and similar for $\psi$ ), then $\hat{\varphi}, \hat{\psi}$ are continuous functions over $\mathbf{R}$ with limit zero at $\pm \infty$ and we have

$$
\hat{\omega}(\xi)=(\varphi * \psi \hat{)}(\xi)=\hat{\varphi}(\xi) \cdot \hat{\psi}(\xi)
$$

But $C_{0}^{\infty}(\mathbf{R}) \subset \mathfrak{L}^{1}(\mathbf{R})$ is a dense subspace of the Hilbertspace $\Re=\mathscr{L}^{2}(\mathbf{R})$ and $F \mid C_{0}^{\infty}(\mathrm{R})$ induces a unitary operator on $\Omega$ (due to Parseval's relation). Accordingly the assignment $u \varepsilon C_{0}^{\infty}(\mathbf{R}) \rightarrow \varphi * u$ defines a bounded operator $(\varphi *)$ of $\Omega$ to itself, with operator norm

$$
\|(\varphi *)\|=\sup _{\|u\|=1}\|(\varphi *) u\|=\operatorname{Max}_{\xi \varepsilon \Re}|\hat{\varphi}(\xi)|=\|\hat{\varphi}\|_{0} .
$$

In the following a bounded operator always will be considered to have domain equal to the whole space, but it evidently suffices to define such operator only for a dense subspace (like $C_{0}^{\infty}$ ) to characterise it.

In the present context we are interested in similar operators acting on the Hilbertspace $\mathfrak{S}=\mathfrak{L}^{2}\left(\mathbf{R}^{+}\right), \mathbf{R}^{+}$denoting the positive real axis.

Definition 1.1. A Wiener-Hopf convolution operator (abbreviated "WHC") is an integral operator over $\mathfrak{S}$ of the form

$$
H_{\varphi} u=(2 \pi)^{-1 / 2} \int_{0}^{\infty} \varphi(x-y) u(y) d y, \quad u \varepsilon C_{0}^{\infty}\left(\mathbf{R}^{+}\right),
$$

with a function $\varphi(s) \varepsilon \mathscr{L}^{1}(\mathbf{R})$.

Relation (1.5) indeed defines a bounded operator of $\mathfrak{S}$. For with the extension operator $E_{n}: \mathfrak{S} \rightarrow \Omega$, defined by

$$
E_{n} u=u(x) \text { in } \mathbf{R}^{+},=0 \text { in } \mathbf{R}-\mathbf{R}^{+}
$$

we get

$$
H_{\varphi}=E_{n}^{*}(\varphi *) E_{n}
$$

especially because $E_{n}^{*}: \Omega \rightarrow \mathfrak{S}$ is the restriction to $\mathbf{R}^{+}$:

$$
E_{n}^{*} u=u \mid \mathbf{R}^{+}, \quad u \varepsilon \mathfrak{L}^{2}(\mathbf{R})
$$

and because $E_{n}, E_{n}^{*}$ are partial isometries. Specifically we have

$$
\left\|H_{\varphi}\right\| \leqq\|\hat{\varphi}\|_{0}
$$

In addition to the operators $H_{\varphi}$ above we also consider $H_{\varphi}{ }^{+}$, defined by

$$
H_{\varphi}^{+} u=(2 \pi)^{-1 / 2} \int_{0}^{\infty} \varphi(x+y) u(y) d y, \quad u \varepsilon C_{0}^{\infty}\left(\mathbf{R}^{+}\right) .
$$

Let $\mathfrak{R}(\mathfrak{S})$ and $\mathfrak{S}(\mathfrak{S})$ denote the algebra of all bounded linear operators, and 
the ideal of all compact (completely continuous) operators of the Hilbert space $\mathfrak{S}$, respectively.

Lemma 1.1. For any $\varphi \varepsilon \mathcal{L}^{1}(\mathbf{R})$ we have $H_{\varphi}{ }^{+}$in $\mathfrak{S}(\mathfrak{S})$.

Proof. First notice that $H_{\varphi}{ }^{+}$is an integral operator with bounded Schmidtnorm (and hence compact) if only $s^{1 / 2} \varphi(s) \varepsilon \mathscr{L}^{2}(\mathbf{R})^{+}$. But the space of all such $\varphi(s)$ is dense in $\mathscr{L}^{1}\left(\mathbf{R}^{+}\right)$, and by Schur's criterion it follows that

$$
\left\|H_{\varphi}^{+}\right\| \leqq \int_{0}^{\infty}|\varphi(s)| d s .
$$

Since $\mathbb{C}$ is closed, the result $H^{+} \varepsilon \subseteq$ for all $\varphi \varepsilon \mathscr{L}^{1}(\Re)$ follows.

Q.E.D.

Let us define two more extension operators $E_{e}$ and $E_{o}$ which associate to any function $u(x) \varepsilon \mathfrak{S}$ the even and odd extension of $2^{-1 / 2} u(x)$ to $\mathrm{R}$, respectively:

$$
\begin{aligned}
& E_{e} u=2^{-1 / 2} u(x), \quad x \varepsilon \mathrm{R}^{+}, \quad=2^{-1 / 2} u(-x), \quad x \varepsilon \mathrm{R}-\mathrm{R}^{+} \\
& E_{o} u=2^{-1 / 2} u(x), \quad x \varepsilon \mathrm{R}^{+}, \quad=-2^{-1 / 2} u(-x), \quad x \varepsilon \mathrm{R}-\mathrm{R}^{+} .
\end{aligned}
$$

We confirm that $E_{e}^{*}$ and $E_{o}^{*}$ are given by

$$
E_{e}^{*} u=2^{1 / 2} u_{e}\left|\mathbf{R}^{+}, E_{o}^{*} u=2^{1 / 2} u_{0}\right| \mathbf{R}^{+},
$$

where $u_{e}$ and $u_{o}$ denote the even and odd part of $u$, defined by

$$
u_{e}(x)=\frac{1}{2}(u(x)+u(-x)), \quad u_{o}(x)=\frac{1}{2}(u(x)-u(-x)) .
$$

Accordingly it follows that

$$
E_{e}^{*} E_{e}=E_{o}^{*} E_{o}=I
$$

and that

$$
\begin{array}{ll}
E_{e} E_{e}^{*} u=u & \text { for even functions, } \\
E_{o} E_{o}^{*} u=u & \text { for odd functions. }
\end{array}
$$

We discriminate between even and odd Wiener-Hopf convolutions (EWHC and $\mathrm{OWHC}$ ), defined as operators $H_{\varphi}$ with $\varphi(s)$ even or odd, respectively. Since the Fourier transform of an even (odd) function is even (odd), it follows that for an even $\varphi$ we get $\varphi * u$ even, whenever $u$ is even. Accordingly we conclude that

$$
H_{\varphi}^{*}=H_{\varphi}+H_{\varphi}^{+}=E_{e}^{*}(\varphi *) E_{e}, \text { for even } \varphi \varepsilon \mathcal{L}^{1}(\mathbf{R}) .
$$

Lemma 1.2. The operators $H_{\varphi}^{*}, \varphi \varepsilon \mathcal{L}^{1}(\Re), \varphi$ even, form a commutative algebra over $\mathbf{C}$ invariant under the involution map * :A $\rightarrow A^{*}$, defined by taking adjoints. Specifically

$$
\begin{aligned}
H_{\varphi}^{*} H_{\psi}^{*} & =H_{\varphi * \psi}^{*}, \\
c H_{\varphi}^{*}+c^{\prime} H_{\psi}^{*} & =H_{c \varphi+c^{\prime} \psi}^{*}, \\
\left(H_{\varphi}^{*}\right)^{*} & =H_{\varphi *}^{*}, \quad \varphi^{*}(x)=\bar{\varphi}(-x) .
\end{aligned}
$$


Proof. Apply (1.16) and (1.17) to obtain the first formula of (1.18). All other statements are then evident.

The commutative $C^{*}$-algebra obtained by adjoining the identity and closing (under operator norm) of the algebra of operators $H_{\varphi}^{*}, \varphi \varepsilon \mathcal{L}^{1}(\mathbf{R})$ will be denoted by $\mathfrak{W}$, and will be called the Wiener-Hopf-algebra.

Let now $F_{c}=E_{e}^{*} F E_{e}$, and notice that $F_{c}^{*} F_{c}=F_{c} F_{c}^{*}=I$, or that $F_{e}$ is a unitary operator of $\mathfrak{S}$. Explicitly we get

$$
F_{c} u=(2 / \pi)^{1 / 2} \int_{0}^{\infty} u(x) \cos x \xi d x=F_{c}^{*} u, \quad u \varepsilon C_{0}^{\infty}\left(\mathbf{R}^{+}\right)
$$

In other words $F_{c}$ is the Fourier-cosine transform. If for any even function $\varphi$ we set $\tilde{\varphi}=\hat{\varphi} \mid \mathrm{R}^{+}=F_{c} \varphi$, it follows at once that

$$
H_{\varphi}^{*}=F_{c}^{*}(\tilde{\varphi}) F_{c} .
$$

We summarize as follows,

Theorem 1.1. (1) The Wiener-Hopf-algebra $\mathfrak{W}$ has its Gelfand space $\mathfrak{T}(\mathfrak{W})$ equal to the closed interval $[0, \infty]$ and is diagonalized by the Fourier cosine transform $F_{c}$ as defined in (1.19).

(2) Every EWHC $H_{\varphi}$ with $\mathcal{L}^{1}$-kernel differs from an element in $\mathfrak{W}$ only by a compact operator.

(3) We have equality in (1.9).

Proof is evident after the above.

2. Mellin-convolutions. While in Section 1 we obtained an algebra which contained (up to a compact additive term) all even Wiener-Hopf convolutions with $\mathscr{L}^{1}$-kernels, we now direct our attention to the specific odd Wiener-Hopfconvolution $H_{1 / x}$ and the commutative $C^{*}$-algebra generated by it.

Define the operators $K_{ \pm}$by setting

$$
K_{ \pm} u=\int_{0}^{\infty} \frac{u(y)}{x \pm y} d y, \quad u \varepsilon C_{0}^{\infty}\left(\mathrm{R}^{+}\right)
$$

where the principal value $\lim _{\epsilon \rightarrow 0} \int_{|x-y| \leqq \epsilon}$ is to be taken in case of $K_{-}$.

We claim that $K_{ \pm}$both are bounded operators of $\mathfrak{S}$. This is immediate, if we use the isometric isomorphism

$$
U: \mathfrak{S} \rightarrow \Re
$$

defined by

$$
u(x) \rightarrow 2^{1 / 2} e^{t} u\left(e^{2 t}\right)=w(t), t \varepsilon \mathbf{R}
$$

which has the property that

$$
\begin{aligned}
& U K_{-} U^{*}=(2 \pi)^{1 / 2}\left((\sinh x)^{-1} *\right), \\
& U K_{+} U^{*}=(2 \pi)^{1 / 2}\left((\cosh x)^{-1} *\right),
\end{aligned}
$$


the right hand side denoting convolution operators, as in (1.1).

Specifically

$$
\begin{aligned}
& (2 \pi)^{1 / 2}(1 / \sinh x)^{\wedge}=-\pi i \tanh t \pi / 2 \\
& (2 \pi)^{1 / 2}(1 / \cosh x)^{\wedge}=\pi /(\cosh t \pi / 2)
\end{aligned}
$$

Accordingly,

$$
\begin{aligned}
& M K_{-} M^{*}=-\pi i \tanh (t \pi / 2), \\
& M K_{+} M^{*}=\pi(\cosh (t \pi / 2))^{-1},
\end{aligned}
$$

with $M=F U$ and with the right hand side denoting the multiplication operator.

Explicitly we get $M$ equal to the Mellin transform $M: \Re \rightarrow \mathfrak{S}$, defined by

$$
u(x) \rightarrow v(t)=2^{-1} \pi^{-1 / 2} \int_{0}^{\infty} u(y) y^{-(1+i t) / 2} d y, \quad u \varepsilon C_{0}^{\infty}\left(\mathbf{R}^{+}\right) .
$$

Definition 2.1. The commutative $C^{*}$-algebra with unit, generated by $K_{-}$above is denoted by $\mathfrak{M}$ and called the algebra of generalized Mellin-convolutions.

It is clear that $K_{+}$and $K_{-}$both belong to $\mathfrak{M}$, and that the Gelfand space of $\mathfrak{T}$ is the interval $[-\infty,+\infty]$, with the Gelfand isomorphism explicitly given by

$$
A \rightarrow M^{*} A M=\varphi_{A}(t),
$$

the right hand side denoting the multiplication operator on $\Omega$.

$\mathfrak{M}$ contains an algebra $\mathfrak{M}^{\prime}$ of integral operators $K$ defined by the assignment

$$
u(x) \rightarrow v(x)=\int_{0}^{\infty} w(x / y) u(y) d y / y, \quad u \varepsilon C_{0}^{\infty}\left(\mathbf{R}^{+}\right),
$$

where $w(x)=\mathcal{L}^{1}\left(\mathbf{R}^{+}, d x / x^{1 / 2}\right)$. Moreover, if we define a modified convolution product $w \odot \chi$ by setting

$$
(w \odot \chi)(x)=\int_{0}^{\infty} w(x / y) \chi(y) d y / y
$$

then $w, \chi \varepsilon \mathscr{L}^{1}\left(\mathbf{R}^{+}, d x / x^{1 / 2}\right)$ implies $w \odot \chi \varepsilon \mathscr{L}^{1}\left(\mathbf{R}^{+}, d x / x^{1 / 2}\right)$, because

$$
\int_{0}^{\infty} d x / x^{1 / 2}\left|\int_{0}^{\infty} w(x / y) \chi(y) d y / y\right|
$$

$$
\begin{aligned}
& \leqq \int_{0}^{\infty}|\chi(y)| d y / y \int_{0}^{\infty}|w(x / y)| d y / x^{1 / 2} \\
& =\left(\int_{0}^{\infty}|\chi(y)| d y / y^{1 / 2}\right)\left(\int_{0}^{\infty}|w(x)| d x / x^{1 / 2}\right) .
\end{aligned}
$$

The product (2.10) is the convolution product of the abelian topological group $\mathbf{R}^{+}$with multiplication as group operation, and the linear function space $\mathcal{L}^{1}\left(\mathbf{R}^{1}, d x / x^{1 / 2}\right)$ becomes a commutative algebra with $\odot$, which is not the group algebra, however, because the Haar measure is given by $d x / x$, not $d x / x^{1 / 2}$. 
With the isometry $U: \mathfrak{S} \rightarrow \Re$ of (2.3) we get

$$
U K_{w} U^{*}=2(2 \pi)^{1 / 2}\left(\left(e^{s} w\left(e^{2 s}\right)\right) *\right)
$$

and

$$
\int_{-\infty}^{+\infty}\left|e^{s} w\left(e^{2 s}\right)\right| d s=\frac{1}{2} \int_{0}^{\infty}|w(t)| d t / t^{1 / 2}<\infty
$$

This shows that $K_{w}$ is a bounded operator in $\mathfrak{M}$, for every $w \varepsilon \mathcal{L}^{1}\left(\mathbf{R}^{+}, d x / x^{1 / 2}\right)$, because the Fourier transform of $e^{s} w\left(e^{2 s}\right)$ is a bounded continuous function in $[-\infty,+\infty]$. Accordingly $\mathfrak{M}^{\prime}$, as defined above, is a subalgebra of $\mathfrak{M}$, and $w \rightarrow$ $K_{w}=(w \odot)$ defines a continuous map from the Banach algebra $\mathscr{L}^{1}\left(\mathrm{R}^{+}, d x / x^{1 / 2}\right)$ onto $\mathfrak{M}^{\prime} \subset \mathfrak{M}$.

The operators of $\mathfrak{M}^{\prime}$ will be called Mellin-convolutions (or MC-operators). It is clear that $K_{+}$is an MC-operator, with

$$
\omega_{+}(x)=(x+1)^{-1} \varepsilon \mathcal{L}^{1}\left(\mathbf{R}^{+}, d x / x^{1 / 2}\right) .
$$

Similarly $K_{-}$is formally generated by

$$
\omega_{-}(x)=(x-1)^{-1} \notin \mathscr{L}^{1}\left(\mathbf{R}^{+}, d x / x^{1 / 2}\right) .
$$

Hence $K_{-}$is not in $\mathfrak{M}^{\prime}$, but we shall call operators of this type "singular MCoperators", in view of their property that

$$
\omega_{-}(x) \varepsilon \mathscr{L}^{1}\left(\mathbf{R}^{+}-[1-\epsilon, 1+\epsilon], d x / x^{1 / 2}\right)
$$

for every $0<\epsilon \leqq 1$ and their singularity at $x=1$ of the type generating a singular integral of $K_{\omega_{-}}$.

Note that the algebra $1+\mathfrak{M}^{\prime}$ is dense in $\mathfrak{M}$ under the operator norm and that for $K_{\omega} \varepsilon \mathfrak{M}^{\prime}$ its symbol function $\varphi_{K_{\omega}}(t) \varepsilon C([-\infty,+\infty])$ is given by the formula

$$
\varphi_{K_{\omega}}(t)=2 \pi^{1 / 2} \omega^{\sim}(t),
$$

with $\omega^{\sim}=M \omega$ denoting the Mellin transform of $\omega$, as defined by (2.7). Clearly $M$ plays the role of the Fourier transform of the group $\mathrm{R}^{+}$.

3. Mixing of Wiener-Hopf- and Mellin-convolutions. We notice that the even Wiener-Hopf-operators of the algebra $\mathfrak{W}$ in Section 1 as well as the Mellin colvolutions of Section 2 (i.e. the operators of $\mathfrak{M}$ ) act on the same Hilbert space $\mathfrak{S}=\mathfrak{L}^{2}(\mathbf{R})$. As a third such commutative $C^{*}$-algebra of linear operators on $\mathfrak{S}$ we mention the algebra $\$=C([0, \infty])$ of multiplications by a continuous function over $[0, \infty]$.

Now we mention the following result.

Lemma 3.1. If $A$ and $B$ are operators in $\mathfrak{B}, \mathfrak{M}$ or $\$$ (not necessarily in the same) then

$$
[A, B]=A B-B A \varepsilon \subseteq(\mathfrak{S}) .
$$


As a proof it suffices to show that the three generating operators $H_{0}-|x|, K_{-}$ and $\left(1+x^{2}\right)^{-1}$ have mutually compact commutators. This is easily accomplished by use of the following well known facts

$$
\begin{aligned}
& \left(e^{-|x|} *\right)\left(1+|x|^{2}\right)^{-1} \varepsilon \subseteq(\Omega) \\
& {\left[\left(\frac{1}{x} *\right),\left(1+x^{2}\right)^{-1}\right] \varepsilon \subseteq(\Omega)} \\
& {\left[\left(e^{-|x|} *\right),\left(\frac{1}{x} *\right)\right]=0 .}
\end{aligned}
$$

Lemma 3.1 demonstrated that the $C^{*}$-algebra with an $\mathfrak{A}=\mathfrak{Y}(\mathfrak{M}, \mathfrak{Y}, \mathfrak{Z})$ generated from all the elements of $\mathfrak{M}, \mathfrak{W}, \mathfrak{Z}$ an $\mathfrak{C}(\mathfrak{Y})$ is an algebra with symbol in the sense of [1]. Moreover it is immediate, repeating essentially the conclusions of $\mathrm{E}$. Herman [6], that the following is true.

Lemma 3.2. a) The symbol space $\mathfrak{M}(\mathfrak{H})$ is homeomorphic to a compact subset of the product

$$
[-\infty,+\infty] \times[0, \infty] \times[0, \infty]=Q
$$

of the three Gelfand spaces $\operatorname{Tr}(\mathfrak{M})=[-\infty,+\infty], \mathfrak{M}(\mathfrak{M})=[0, \infty]$ and $\operatorname{Tr}(\Re)=$ $[0, \infty]$, the homeomorphism being established by the product ı of the three associate dual maps

$$
\begin{aligned}
& \pi_{1}^{\prime}: \mathfrak{I T}(\mathfrak{R}) \rightarrow \operatorname{TK}(\mathfrak{M}) \\
& \pi_{2}^{\prime}: \operatorname{IT}(\mathfrak{I}) \rightarrow \operatorname{IN}(\mathfrak{W}) \\
& \pi_{3}^{\prime}: \operatorname{Tr}(\mathfrak{H}) \rightarrow \operatorname{Mr}(\mathfrak{Z})
\end{aligned}
$$

of the natural imbedding

$$
\pi_{1}: \mathfrak{M} \rightarrow \mathfrak{A} / \mathfrak{C}, \quad \pi_{2}: \mathfrak{Y} \rightarrow \mathfrak{U} / \mathfrak{S}, \quad \pi_{3}: \mathfrak{B} \rightarrow \mathfrak{A} / \mathfrak{S},
$$

with $\mathbb{S}=\mathbb{S}(\mathfrak{S})$.

b) If $p_{1}, p_{2}, p_{3}$ denote the natural projections, mapping the triples of the product space onto the first, second and third component, respectively, then the three maps $p_{j} \circ \iota$ are surjective, $j=1,2,3$.

To highlight the proof, notice that assertion b) simply says that the associated maps $\pi_{i}^{\prime}$ are surjective, which for $C^{*}$-algebras follows from the fact that the imbedding homomorphisms $\pi_{i}$ of (3.4) are injective. (None of the algebras $\mathfrak{M}, \mathfrak{B}, \mathbb{Z}$ has more than the zero operator in common with $\mathfrak{E}(\mathfrak{S})$. For assertion a) let $m_{0}, m_{1} \varepsilon \mathfrak{N}(\mathfrak{U})$ have the same image under $\iota$, then for any operator $A \varepsilon \mathfrak{M}$, $\mathfrak{W}$ or $\$$ we have

$$
\begin{aligned}
\sigma_{A}\left(m_{0}\right) & =\varphi_{A}^{i}\left(\pi_{j}^{\prime} m_{0}\right)=\varphi_{A}^{j}\left(\pi_{i}^{\prime} m_{1}\right) \\
& =\sigma_{A}\left(m_{1}\right),
\end{aligned}
$$


where $\sigma$ denotes the symbol of $A$ in $\mathfrak{A}$ and $\varphi_{A}^{j}(x)$ is the Gelfand isomorphism in $\mathfrak{M}, \mathfrak{W}$ or $\mathbb{Z}, j=1,2,3$, in this order, respectively. This just uses a well known property of the associated dual map. So, since $\mathfrak{A}$ is generated by the elements in $\mathfrak{E}, \mathfrak{M}, \mathfrak{W}$ and $\mathfrak{Z},(3.5)$ implies that $\sigma_{A}\left(m_{0}\right)=\sigma_{A}\left(m_{1}\right)$ for all $A \varepsilon \mathfrak{A}$. Or, we must have $m_{0}=m_{1}$, i.e., ı constitutes a homeomorphism.

Q.E.D.

From now on in this section we will discriminate between the points in $\mathscr{T}(\mathfrak{M})$, $\operatorname{Tr}(\mathfrak{B})$ and $\mathscr{T}((3)$ by using the symbols $t, \xi$ and $x$, respectively.

After Lemma 3.2 the problem of characterising the symbol space of $\mathfrak{A}$ is reduced to the question of determining the precise subset of the cube (3.2) onto which it is mapped. For the following we shall always identify this subset with the symbol space via the homeomorphism $\iota$. First we note the following result.

Lemma 3.3. Let $A \varepsilon \mathfrak{M}, B \varepsilon \mathfrak{W}, C \varepsilon \Re$ be given. Then the product $A \cdot B \cdot C$ is $a$ compact operator, if and only if the symbol space $\mathfrak{T}(\mathfrak{U})$ is a subset of the set

$$
\left\{(t, \xi, x): \varphi_{A}^{1}(t) \cdot \varphi_{B}^{2}(\xi) \cdot \varphi_{C}^{3}(x)=0\right\}
$$

Proof. We will have

$$
\sigma_{A B C}=\left(\varphi_{A}^{1}(t) \cdot \varphi_{B}^{2}(\xi) \cdot \varphi_{C}^{3}(x)\right) \mid \operatorname{Tr}(\mathfrak{H})
$$

for any product $A B C$ of $A \varepsilon \mathfrak{M}, B \varepsilon \mathfrak{W}, C \varepsilon \Re$. Since $A B C$ is compact if and only if $\sigma_{A B C}=0$, the statement is implied.

Q.E.D.

Any unitary operator $S$ which leaves all three algebras invariant:

$$
S^{*} A S, S A S^{*} \varepsilon \mathfrak{M}, \mathfrak{W}, \mathfrak{Z} \text { for } A \varepsilon \mathfrak{M}, \mathfrak{W}, \mathbb{B} \text { (respectively), }
$$

will also leave $\mathfrak{A}$ invariant and therefore induce homeomorphisms of all 4 Gelfand spaces (defined as associated dual map of the induced isomorphisms $\mathfrak{M} \rightarrow \mathfrak{M}$, etc.).

While Lemma 3.3 may be used to restrict the set constituting $\mathfrak{T}(\mathfrak{A})$ the last remark will help to establish its extent. The result is as follows.

Theorem 3.1. a) $\mathfrak{T}(\mathfrak{A})$ is identical with the subset of $Q$ described in Fig. 1. In details, this subset is characterised as follows:

(i) Over $x=0$ there is precisely the segment $\xi=\infty,-\infty \leqq t \leqq+\infty$ in $\mathfrak{T}(\mathfrak{U})$.

(ii) Over any $x \neq 0, \infty$ there are just the two points $\xi=\infty, t= \pm \infty$ in the set $\mathfrak{T}(\mathfrak{A})$.

(iii) Over $x=+\infty$ there are the two segments $0 \leqq \xi \leqq \infty, t= \pm \infty$ and the segment $\xi=0,-\infty \leqq t \leqq+\infty$ in $\operatorname{Tr}(\mathfrak{H})$.

b) For any finitely generated element

$$
A=\sum_{i=1}^{N} A_{i}^{1} A_{i}^{2} A_{i}^{3}+C, \quad C \varepsilon \mathfrak{s}, \quad A_{j}^{1} \varepsilon \mathfrak{M}, \quad A_{i}^{2} \varepsilon \mathfrak{W}, \quad A_{i}^{3} \varepsilon \Re
$$

we get

$$
\sigma_{A}(t, \xi, x)=\sum_{j=1}^{N} \varphi_{A^{1}}^{1}(t) \varphi_{A^{2}}^{2}(\xi) \varphi_{A^{3}}^{3}(x) \quad \text { for } \quad(t, \xi, x) \varepsilon \mathfrak{M}(\mathfrak{R})
$$




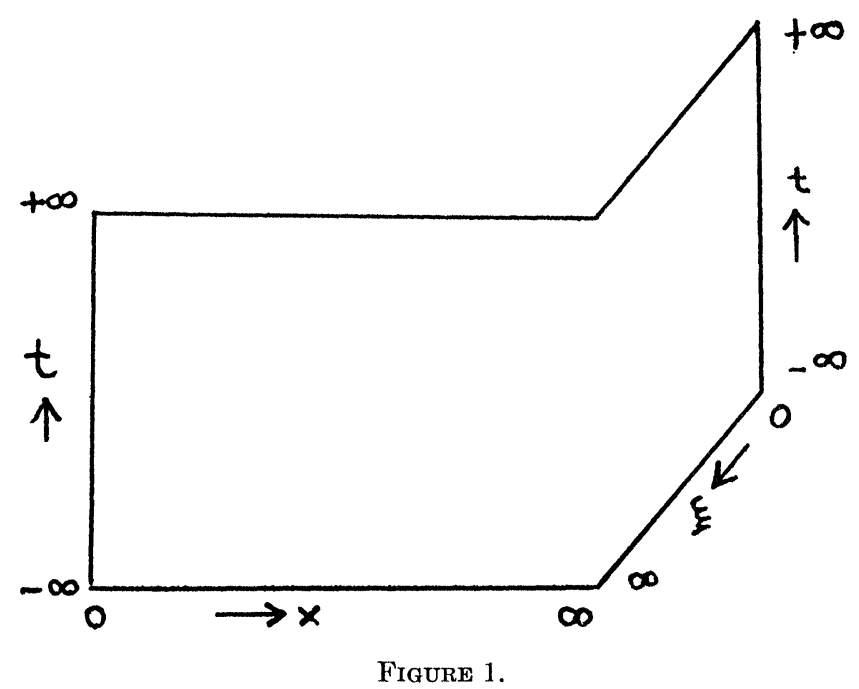

Proof. The following facts are to be verified.

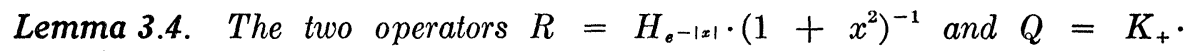
$x\left(1+x^{2}\right)^{-1}$ are compact.

Both these statements are easy consequences of well known facts on integral operators.

Next we require the following result, which is somewhat less familiar.

Lemma 3.5. Let $\psi(x) \varepsilon \mathscr{L}^{1}(\mathbf{R})$ be defined by

$$
\hat{\psi}(\xi)=\frac{1+2 \xi^{2}}{\left(1+\xi^{2}\right)^{2}}, \quad \xi \varepsilon \mathbf{R}
$$

and let

$$
\omega(x)=x^{-1 / 2} e^{-(\log x)^{2}}, \quad x \varepsilon \mathbf{R}^{+}
$$

and

$$
\chi(x)=e^{-1 / x^{2}}, \quad x \varepsilon \mathrm{R}^{+} .
$$

Then we have

$$
P=\chi K_{\omega}\left(1-H_{\psi}\right) \chi \varepsilon \mathfrak{S}(\mathfrak{S}) .
$$

Proof. One first confirms that $P$ is an integral operator with kernel $p(x, y)$, where

$$
p(x, y)=\chi(x) \chi(y)\left[(1 / y) \omega(x / y)-(2 \pi)^{-1 / 2} \int_{0}^{\infty}(1 / t) \omega(x / t) \psi(y-t) d t\right]
$$


Define a function $\gamma(t)$ by

$$
\gamma(t)=\omega(1 / t) / t \text { for } t>0,=0 \text { for } t \leqq 0,
$$

and notice that $\gamma(t) \varepsilon S$, where $S$ denotes the space of all $C^{\infty}(\mathbf{R})$-functions vanishing at $\pm \infty$ together with all derivatives stronger than any power of $t$. Using (3.12) together with well known facts on Fourier transforms and convolution products we get

$$
p(x, y)=(2 \pi)^{-1 / 2} \chi(x) \chi(y) \int_{-\infty}^{+\infty} \hat{\gamma}(\eta x) \frac{\eta^{4}}{\left(1+\eta^{2}\right)^{2}} e^{i \eta y} d y
$$

A 2-fold integration by parts then yields

$$
p(x, y)=c \chi(x) \chi(y) y^{-2} \int_{-\infty}^{+\infty} \frac{\partial^{2}}{\partial \eta^{2}}\left(\gamma^{\hat{n}}(\eta x) \frac{\eta^{4}}{\left(1+\eta^{2}\right)^{2}}\right) e^{i \eta y} d \eta .
$$

Also we may estimate the integral in (3.18) by a sum of 3 terms

$$
x^{k} \int_{-\infty}^{+\infty}\left|\delta_{k}(\eta x)\right||\eta|^{2+k} d \eta, \quad k=0,1,2
$$

where $\delta_{k}(\eta), k=0,1,2$, denote functions in $\delta$. Also (3.19) equals $c_{k} x^{-3}$ with

$$
c_{k}=\int_{-\infty}^{+\infty}\left|\delta_{k}(t)\right| t^{2+k} d t .
$$

Accordingly we get

$$
p(x, y) \leqq c\left(\chi(x) / x^{3}\right)\left(\chi(y) / y^{2}\right) .
$$

This shows that $P$ is a compact operator.

Q.E.D.

Coming back to the proof of Theorem 3.1., we notice that the operators $K_{+}$, $H_{e^{-|x|}}, K_{\omega}, 1-H_{\psi}$ correspond to the functions $\pi / \cosh (t \pi / 2),(2 / \pi)^{1 / 2}(1+$ $\left.\xi^{2}\right)^{-1},(2 / \pi)^{1 / 2} e^{-t^{2} / 4}$ and $\xi^{4} /\left(1+\xi^{2}\right)^{2}$, respectively. Using the Lemmata $3.3,3.4$ and 3.5 it then is evident that the space described in Theorem 3.1 does contain the symbol space $\mathfrak{T}(\mathfrak{A})$.

We will postpone verification of the reverse inclusion until a later section, where we will have other algebras on hand.

4. The operator $\mathbb{K}_{ \pm}^{0}$. In this section we study the pair of integral operators $K_{ \pm}^{0}: \mathfrak{S} \rightarrow \mathfrak{S}$ defined by

$$
u(x) \rightarrow v(x)=\int_{\mathfrak{Y}^{+}} e^{-|x \neq y|} u(y) d y /(x \pm y) .
$$

(with a singular integral in case of $K_{-}^{0}$.) It will be our intention to study the $C^{*}$-algebra $\mathfrak{A}^{0}$ generated by $K_{ \pm}^{0}, \mathfrak{W}, \mathfrak{3}$ and $\mathfrak{C}(\mathfrak{S})$. This algebra will be shown to be a subalgebra of $\mathfrak{A}$, with slightly more natural properties in view of differential operators. 
First we need a different representation of $K_{ \pm}^{0}$.

Lemma 4.1. Let the function $\psi_{0}(x)$ be defined by

$$
\psi_{0}(x)=(2 \pi)^{1 / 2} \int_{0}^{\infty} \sin t d t /\left(x^{2}+t^{2}\right), \quad x \varepsilon \mathbf{R} .
$$

Then the even function $\psi_{0}(x)$ is in $\mathcal{L}^{1}(\mathbf{R}) \cap C^{\infty}\left(\mathbf{R}^{+}\right)$and we have

$$
K_{ \pm}^{0}=\left(1-2 / \pi^{2} H_{\psi_{0}}\right) K_{ \pm}+C_{ \pm} \text {with } C_{ \pm} \varepsilon(5(\mathfrak{S}) .
$$

Proof. We first show that $\psi_{0} \varepsilon C^{\infty}\left(\mathbf{R}^{+}\right)$by differentiating under the integral sign and that

$$
\psi_{0}(x)=O(\log |x|) \text { at } \quad x=0
$$

and

$$
\psi_{0}(x)=O\left(x^{-2}\right) \text { at } x=\infty
$$

(by a suitable partial integration). This confirms that $\psi_{0} \varepsilon \mathscr{L}^{1}(\mathrm{R})$.

Next we claim that we have

$$
\left(e^{-|x|} / x^{*}\right)=\left(1 / x^{*}\right)\left(1-2 / \pi^{2}\left(\psi_{0} *\right)\right)
$$

with convolution products as in (1.1), and with the convolution integrals interpreted as principal values, wherever needed.

Indeed we confirm by standard methods that

$$
(1 / x *)\left(\psi_{0} *\right)=\omega_{0} *
$$

with

$$
\omega_{0}(x)=\int_{0}^{\infty} \sin t d t \int_{-\infty}^{+\infty}\left((y-x)^{2}+t^{2}\right)^{-1} d y / y
$$

the integrals being principal values if needed. By a complex integral method one confirms that

$$
\text { p.v. } \int_{R}\left((y-x)^{2}+t^{2}\right)^{-1} d y / y=\pi x / t\left(x^{2}+t^{2}\right)^{-1} .
$$

Or, we get

$$
\begin{aligned}
\omega_{0}(x) & =\pi x \int_{\mathbf{R}^{+}}\left(x^{2}+t^{2}\right)^{-1} \sin t d t / t \\
& =\pi / 2 x \operatorname{Im} \text { p.v. } \int_{\mathbf{R}}\left(x^{2}+t^{2}\right)^{-1} e^{i t} d t / t \\
& =\pi^{2} / 2\left(1 / x-e^{-|x|} / x\right)
\end{aligned}
$$

by evaluating a second complex integral. Then (4.7) and (4.10) imply (4.6).

To conclude the statement of the lemma, we now apply the method of Section 
1, involving the even and odd extension operators $E_{e}$ and $E_{o}$. It is clear from (1.17) that

$$
\begin{aligned}
& E_{e}^{*}\left(\psi_{0}^{*}\right) E_{e}=H_{\psi_{0}}+H_{\psi_{0}}^{+}, \\
& E_{o}^{*}\left(\psi_{0} *\right) E_{o}=H_{\psi_{0}}-H_{\psi_{0}}^{+} .
\end{aligned}
$$

On the other hand the convolutions $\left(1 / x^{*}\right)$ and $\left(e^{-|x|} / x^{*}\right)$ are both odd, so that even functions are converted into odd functions and vice versa. Hence we get

$$
\begin{aligned}
E_{o}^{*}\left(1 / x^{*}\right) E_{e} & =K_{-}+K_{+}, \\
E_{o}^{*}\left(e^{-|x|} / x^{*}\right) E_{e} & =K_{-}^{0}+K_{+}^{0}, \\
E_{e}^{*}\left(1 / x^{*}\right) E_{o} & =K_{-}-K_{+}, \\
E_{e}^{*}\left(e^{-|x|} / x^{*}\right) E_{o} & =K_{-}^{0}-K_{+}^{0} .
\end{aligned}
$$

We know from Lemma 1.1 that $H_{\psi_{0}}^{+} \varepsilon$ $(\mathfrak{S})$. Accordingly from (4.11), (4.12) and (1.16) we get the desired relation (4.3).

Q.E.D.

It is evident after Lemma 4.1 that $K_{ \pm}^{0}$ are in $\mathfrak{A}$ of Section 3. To compute their symbol we must obtain the Fourier transform of the function $\psi_{0}$ of (4.2).

Lemma 4.2. We have

$$
\hat{\psi_{0}}(x)=\pi(\pi / 2-\arctan |x|)
$$

Proof. One computes directly that

$$
\hat{\psi_{0}}(x)=\pi \int_{0}^{\infty} \sin t e^{-t|x|} d t / t
$$

and then may evaluate the integral to get the desired result.

Lemma 4.3. We have $K_{ \pm}^{0} \varepsilon \mathfrak{A}$ and

$$
\begin{aligned}
& \sigma_{K-\circ}(t, \xi, x)=-2 i \arctan \xi \tanh (t \pi / 2), \\
& \sigma_{K+}(t, \xi, x)=2 \arctan \xi / \cosh (t \pi / 2),
\end{aligned}
$$

for $(t, \xi, x) \varepsilon \mathfrak{T l}(\mathfrak{U})$.

The proof of Lemma 4.3 is a consequence of Lemma 4.2 and formulas (2.6).

Concerning the algebra $\mathfrak{P}^{0}$, defined initially in this section, we now have the following result.

Theorem 4.1. We have $\mathfrak{U}^{0} \subset \mathfrak{H}$ (properly), and an operator $A \varepsilon \mathfrak{A}$ is in $\mathfrak{A}^{0}$ if and only if its symbol is constant on the segment

$$
x=\infty, \quad \xi=0, \quad-\infty \leqq t \leqq+\infty
$$

of the symbol space $\mathfrak{T}(\mathfrak{H})$.

The symbol space $\mathfrak{T}\left(\mathfrak{H}^{0}\right)$ arises from $\mathfrak{T (}(\mathfrak{U})$ by identifying the points of the segment (4.16). 
The proof of Theorem 4.1 is a consequence of the Stone-Weierstrass theorem, noting that $\sigma_{K \pm 0}$ do not separate the points of the segment (4.16).

5. Other subalgebras of $\mathfrak{A}$. In this section we want to discuss two other interesting subalgebras of $\mathfrak{A}$. The first already was discussed in [6] and was called $\mathfrak{B}$ there. It is defined as the $C^{*}$-algebra (with unit) generated by the elements of $\mathfrak{M}, \mathbb{B}$ and $\mathbb{C}$.

In [6] it was seen that the symbol space $\mathfrak{T}(\mathfrak{B})$ is homeomorphic to the boundary of the square $\operatorname{Tr}(\Re) \times \operatorname{Tr}(\mathfrak{M})$. There we also obtained an index formula saying that for $A \varepsilon \mathfrak{B}$ the Fredholm index

$$
\text { ind } A=\operatorname{dim} \operatorname{ker} A-\operatorname{codim} \operatorname{im} A
$$

is equal to the winding number of the symbol $\sigma_{\boldsymbol{A}}$.

We use both facts to complete the proof of Theorem 3.1. There it still was left to be shown that $\mathfrak{T}(\mathfrak{H})$ contains all points of the set described in the figure. Now, if we note that the associated dual map $\pi^{\prime}$ of the imbedding

$$
\pi: \mathfrak{P} / \mathfrak{S} \rightarrow \mathfrak{U} / \mathfrak{C}
$$

is surjective and coincides with the natural projection

$$
(\xi, t, x) \rightarrow(t, x)
$$

it becomes clear that $\mathfrak{T}(\mathfrak{U})$ must contain the segments

$$
x=0, \quad \xi=\infty, \quad-\infty \leqq t \leqq+\infty
$$

and

$$
0 \leqq x \leqq \infty, \quad \xi=\infty, \quad t= \pm \infty
$$

and

$$
x=\infty, \quad \xi=0, \quad-\infty \leqq t \leqq+\infty .
$$

As to the remaining two segments first notice that the unitary operator $W_{\alpha}$ : $\mathfrak{S} \rightarrow \mathfrak{S c}$ defined by

$$
u(x) \rightarrow \alpha^{1 / 2} u(\alpha x)=v(x)
$$

leaves $\mathfrak{M}, \mathfrak{W}$, and $§$ invariant, for any positive constant $\alpha$. We compute that

$$
A \rightarrow W_{\alpha}^{*} A W_{\alpha}
$$

induces the homeomorphism

$$
(t, \xi, x) \rightarrow(t, \xi / \alpha, \alpha x)
$$

in $\mathfrak{T}(\mathfrak{U})$. Hence, if any interior point of the segment

$$
x=\infty, \quad 0 \leqq \xi \leqq \infty, \quad t=\infty
$$

is in $\mathfrak{T}(\mathfrak{U})$ then the whole segment must be in $\mathfrak{T}(\mathfrak{U})$. Similarly with

$$
x=\infty, \quad 0 \leqq \xi \leqq \infty, \quad t=-\infty .
$$


It only can be that both these segments are entirely in $\mathfrak{T}(\mathfrak{H})$. For otherwise (every component of) $\mathfrak{T}(\mathfrak{A})$ would be contractible which means that every operator in $\mathfrak{A}$ has Fredholm index zero, while we know that there are elements in $\mathfrak{B} \subset \mathfrak{A}$ with index $\neq 0$. Therefore Theorem 3.1 is established.

Following this last argument we also get the following result.

Theorem 5.1. The index of a Fredholm operator $A$ in $\mathfrak{A}$ is equal to the winding number of the symbol $\sigma_{A}$ on $\mathfrak{T}(\mathfrak{A})$ which is homeomorphic to a circle, with the orientation coinciding with the orientation of increasing $x$ on the segment $t=-\infty$, $\xi=\infty, 0 \leqq x \leqq \infty$.

For application in a later paper (See [3]) we also consider the algebra $\mathfrak{F}$ with symbol generated from $K_{ \pm}^{\circ}, \mathfrak{W}$ and $\mathfrak{C}$. First we find that Lemma 4.3 results in the relation

$$
\sigma_{A^{0}}=4 / \pi^{2} \text { arc } \tan ^{2} \xi, \text { for } A^{0}=\left(K_{+}^{0}\right)^{2}-\left(K_{-}^{0}\right)^{2},
$$

which shows that the operator $A^{0}$ generates the algebra $\mathfrak{W}$ modulo $\mathfrak{S}$. Hence $\mathfrak{F}$ is generated by $K_{ \pm}^{0}$ and $\mathbb{E}$ alone.

Clearly we have

$$
\mathfrak{F} \subset \mathfrak{H}^{0} \subset \mathfrak{A} \text {. }
$$

Theorem 5.2. The symbol space $\mathfrak{T}(\mathfrak{F})$ results from $\mathfrak{M}\left(\mathfrak{A}^{0}\right)$ by identifying the points of the two segments

$$
\xi=\infty, \quad t= \pm \infty, \quad 0 \leqq x \leqq \infty
$$

each. It is homeomorphic to a circle. If it is given the orientation induced by $\mathfrak{T}(\mathfrak{A})$, then the index of a Fredholm operator is equal to the winding number of its symbol.

Corollary to Theorem 4.1. $\operatorname{Tr}\left(\mathfrak{A}^{0}\right)$ is homeomorphic to a circle. If given the orientation of $\mathfrak{T}(\mathfrak{U})$ the index of a Fredholm operator is equal to the winding number of the symbol.

6. Pseudo-differential operators on $\mathrm{R}^{+}$. In [5], Section 11,12 , jointly with E. Herman we were proving Fredholm-type results for an algebra $\mathfrak{B}$ of linear operators on functions over $\mathrm{R}^{n}$. The algebra $\mathfrak{B}$ was shown to contain all pseudodifferential operators over $\mathrm{R}^{n}$ of the type considered by Kohn and Nirenberg [7] (up to an additive term of order $-\infty$ ); we were denoting the elements of $\mathfrak{B}$ as generalized pseudo-differential operators $(G \psi D O)$.

In the following we reanalyze our method of [6] with the purpose of generalizing it to the case of a manifold more general than $\mathbf{R}^{n}$. We then here will apply the principles developed to another very special manifold: the half-line $\mathrm{R}^{+}$, for which several algebras with symbol were studied in the preceding sections.

In defining a $G \psi D O$ (in [6]) we were using

a) an algebra with symbol $\mathfrak{Q}$ over $\mathfrak{S}_{0}=\mathfrak{L}^{2}\left(\mathbf{R}^{n}\right)$;

b) a class of "comparison operators" $\Lambda_{s}$ in $\mathfrak{S}_{0}$ defined by

$$
\Lambda_{s}=(1-\Delta)^{-s / 2},-\infty<s<+\infty,
$$


with $\Delta$ denoting the (unbounded) operator of $\mathfrak{S}_{0}$ characterized as the only self adjoint extension of the Laplace operator $\sum_{i=1}^{n} \partial^{2} / \partial x_{i}^{2}$ in $C_{0}^{\infty}\left(\mathrm{R}^{n}\right)$.

Ignoring some finer details, a $G \psi D O L$ was defined as any product of a comparison operator and an operator in $\mathfrak{D}$ :

$$
L=A \Lambda_{s}, A \varepsilon \mathfrak{D} .
$$

Note that the comparison operators were self-adjoint and positive definite. To each of them we would associate another Hilbert space $\mathfrak{S}_{s}$, just by completing the domain $D\left(\Lambda_{s}\right)$ of $\Lambda_{s}$ under the inner product $(u, v)_{s}=\left(\Lambda_{s} u, \Lambda_{s} v\right)$ and $\Lambda_{s}$ may be reinterpreted as an isometry from $\mathfrak{S}_{s}$ onto $\mathfrak{S}_{0}$.

Now $A \varepsilon \mathfrak{Q}$ is Fredholm if and only if its symbol $\sigma_{A}$ does not vanish. Accordingly, $L$ is Fredholm as an operator from $\mathfrak{S}_{s}$ to $\mathfrak{S}_{0}$ if and only if $\sigma_{A} \neq 0$. In other words, if $L$ is considered to act from $\mathfrak{S}_{s}$ to $\mathfrak{S}_{0}$ then its Fredholm property is well determined by $\sigma_{A}$, the symbol of $A$.

On the other hand, for $s \geqq 0$ the operators $\Lambda_{s}$ are bounded below:

$$
\left\|\Lambda_{s} u\right\| \geqq\|u\|, \quad u \varepsilon \mathfrak{D}\left(\Lambda_{s}\right) .
$$

Accordingly, in this case $L$ may be considered as an unbounded operator from $\mathfrak{S}_{0}$ to itself and it will be Fredholm if and only if $\sigma_{A} \neq 0$.

In case of the half line let us consider constant coefficient differential operators with boundary conditions, defined as follows: Let

$$
P(\lambda), B_{1}(\lambda), \cdots B_{r}(\lambda)
$$

denote polynomials in the one variable $\lambda$ with complex coefficients, none of them $\equiv 0$. Let $P(\lambda)$ be of order $N$ and $B_{i}(\lambda)$ of order $s_{i} \leqq N-1, j=1, \cdots, r ; r \leqq N$. Assume that $B_{i}(\lambda), j=1, \cdots, r$, are linearly independent. We then define a differential operator $L$, as an unbounded closed operator of $\mathfrak{S}=\mathfrak{L}^{2}\left(\mathbf{R}^{+}\right)$as follows.

The domain $D(L)$ of $L$ is the collection of all functions $u \varepsilon \mathfrak{S}$ such that

(i) $u, u^{\prime}, u^{\prime \prime}, \cdots, u^{(N-2)}$ are continuous; $u^{(N-1)}$ is absolutely continuous;

(ii) $u, u^{(N)} \varepsilon \mathfrak{S}$;

(iii) $B_{i}(D) u=0, j=1, \cdots, r$, at $x=0$.

Here $u^{(j)}=d^{i} u / d x^{i}, D=-i d / d x$.

Then the operator $L$ is given by

$$
L u=P(D) u, u \varepsilon D(L) .
$$

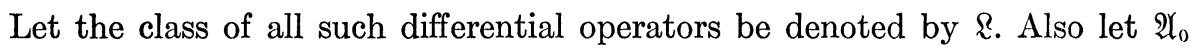
denote the algebra with symbol as introduced in Section 4.

Definition 6.1. An $\mathfrak{A}_{0}-\psi D O$ of type $L$ is an operator of the form

$$
K=A\left(L^{*} L+1\right)^{s / 2}, \quad A \varepsilon \mathfrak{U}_{0}, \quad L \varepsilon \mathfrak{z}
$$

$s$ is called an order of $K$ relative to $L$.

Theorem 6.1. Any differential operator $K$ defined by

$$
K u=K(x, D) u
$$


for $u \varepsilon \mathfrak{D}(K)$, with $D(K)$ characterized as earlier $D(L)$ with a set of boundary conditions (iii), and with $K(x, \lambda)$ a polynomial in $\lambda$ with coefficients being continuous functions of $x$ in $[0, \infty]$ is an $\mathfrak{H}_{0}-\psi D O$ of type $L$ and of order one where the constant coefficients differential operator $L$ is determined by

$$
P(D)=K(0, D)
$$

with the same boundary condition polynomials than $K$, provided that the order of $K(x, D)$ at $x$ is never larger than that of $P(D)=K(0, D)$.

For the proof of this result we merely must show that all operators

$$
D_{i}\left(L^{*} L+1\right)^{-1 / 2}, \quad j=0,1, \cdots, N,
$$

are in $\mathfrak{A}_{0}$. Indeed, if this is assumed correct, it follows that

$$
A=K\left(L^{*} L+1\right)^{-1 / 2}=K(x, D)\left(L^{*} L+1\right)^{-1 / 2} \varepsilon \mathfrak{A}_{0},
$$

especially

$$
\mathfrak{D}(K) \subset \mathfrak{D}(L) .
$$

As a consequence we get (6.6) with $s=1$, thereby proving the statement.

The verification of (6.9) to be in $\mathfrak{A}_{0}$ is a rather technical task, yet following well established rules: First one will set up the well known technique of inverting the operator $\left(L^{*} L+1-\lambda\right)=R_{\lambda}$ for suitable complex $\lambda$, which leads into Lopatinsky type of arguments. Then one obtains the square root by the well known resolvent integral

$$
\left(L^{*} L+1\right)^{-1 / 2}=-\frac{1}{2 \pi i} \int_{\Gamma} R_{\lambda} \lambda^{-1 / 2} d \lambda
$$

over a suitable path $\Gamma$, for instance the straight line $\operatorname{Re} \lambda=\frac{1}{2}$.

One thus obtains explicit control of the operators (6.9) and is able to show that these are integral operators in $\mathfrak{A}_{0}$, as stated.

We will leave the detailed discussion of these facts to another publication.

\section{LITERATURE}

[1] M. Breuer \& H. O. Cordes, On Banach algebras with $\sigma$-symbol, J. Math. Mech., 13 (1964) 313-324.

[2] - Part II, J. Math. Mech., 14 (1965) 299-314.

[3] H. O. Condes, An algebra of singular integral operators on a half-space, (to appear).

[4] H. O. Cordes \& E. Herman, Singular integral operators on a half-line, Proc. Nat. Acad. Sci., 56 (1966) 1668-1673.

[5] $\longrightarrow$, Remarks on the Gel'fand theory of pseudo-differential operators, Amer. J. Math., (to appear).

[6] E. Herman, The symbol of the algebra of singular integral operators, J. Math. Mech., 15 (1966) 147-156.

[7] J. Kohn \& L. Nirenberg, An algebra of pseudo-differential operators, Comm. Pure Appl. Math., 18 (1965) 269.

For further literature in the general area see the bibliographies of [1], [4] and [5]. 